\title{
Possíveis novos determinantes da qualidade de vida de pacientes com câncer de tireoide tratado: um estudo qualitativo
}

\author{
New possible determinants of the quality of life of patients \\ with treated thyroid cancer: a qualitative study
}

\author{
Carla Maria Ramos Germano ${ }^{1}$ \\ Débora Bonato ${ }^{1}$ \\ Victor Hugo Maion ${ }^{2}$ \\ Lucimar Retto da Silva de Avó ${ }^{1}$ \\ Débora Gusmão Melo ${ }^{1}$ \\ Bruno José Barcellos Fontanella ${ }^{1}$
}

${ }^{1}$ Departamento de Medicina, Centro de Ciências Biológicas e da Saúde, Universidade Federal de São Carlos. Rod. SP-310 Km 235, Campus UFSCAR. 13565-905 São Carlos SP Brasil.cgermano@ufscar.br ${ }^{2}$ Santa Casa de São Carlos. São Carlos SP Brasil.

\begin{abstract}
The aim of this study was to develop a qualitative approach of determinant factors of the quality of life of treated patients with papillary thyroid carcinoma. Sixteen in-depth interviews were conducted with individuals 18 to 45 years old regarding their disease representations and experiences, followed by statements content analysis. The results show issues already provided in structured questionnaires about quality of life, although others were only partially assumed in these surveys (management of the concept of disease etiology; the "forced" patient introduction into the medical conceptual universe; the fear of the prognosis and positive changes in lifestyle). The results interpretation were benefited from recently developed theoretical elaborations: the anxieties related to illness experience seem to be configured as a "modern risk", in the context of a "risk society". The development of structured questionnaires on quality of life requires frequent qualitative studies to capture changes in subjective aspects of the construct, given the dynamic changes of historical, cultural and psychological meanings of the health disease process, constantly influenced by technological innovations and continuing epidemiological interpretations.
\end{abstract}

Key words Qualitative research, quality of life, papillary thyroid carcinoma, adults.
Resumo Objetivou-se uma abordagem qualitativa de fatores que determinariam a qualidade de vida de pacientes com carcinoma papilifero de tireoide tratados. Foram feitas 16 entrevistas em profundidade com indivíduos de 18 a 45 anos sobre suas representações e experiências com esta enfermidade, seguidas por análise de conteúdo de enunciados. Os resultados contêm aspectos já previstos em questionários estruturados sobre qualidade de vida, mas outros estão parcialmente presumidos nesses questionários (manejo do conceito de etiologia da doença, a inserção "forçada" dos pacientes no universo conceitual médico, o medo do prognóstico e as mudanças "positivas" no estilo de vida). A interpretação desses resultados beneficiou-se de elaborações teóricas de desenvolvimento recente: as angústias sobre a experiência da doença parecem configurar-se, para os participantes, como um "risco moderno", no contexto de uma "sociedade de riscos". O desenvolvimento de questionários estruturados de qualidade de vida requer constantes estudos qualitativos que captem mudanças nos aspectos subjetivos do construto, dada a dinamicidade dos significados históricoculturais e psicológicos do processo saúde-doença, constantemente influenciados por inovações tecnológicas e sucessivas interpretações epidemiológicas.

Palavras-chave Pesquisa qualitativa, Qualidade de vida, Carcinoma papilífero da tireoide, Adultos 


\section{Introdução}

A prevalência de câncer da tireoide aumentou nas últimas décadas em todo mundo, inclusive no Brasil, e as causas para este aumento ainda estão em discussão $0^{1,2}$.

O carcinoma papilífero da tireoide (PTC) é responsável por $85 \%$ desses casos de câncer e, embora o PTC possa ocorrer em qualquer idade, sua distribuição temporal mostra um padrão bimodal, com um pico entre 25 e 30 anos e um segundo pico entre 55 e 60 anos $^{3}$. A idade média de diagnóstico é de 44 anos e a incidência entre mulheres é três vezes maior do que entre homens. Em geral, o prognóstico dos pacientes é excelente e a taxa de sobrevivência de um ano é de $93 \%$, apesar desta condição poder retornar muitos anos após o diagnóstico e o tratamento iniciais ${ }^{4,5}$. A expectativa de vida é a mesma que para a população geral, exceto para pacientes idosos com estadiamento avançado ${ }^{6}$.

Tradicionalmente, o sucesso do tratamento de pacientes com câncer tem sido avaliado pelo tempo de sobrevivência e, nesse aspecto, o tratamento do PTC pode ser considerado bem sucedido. Entretanto, como o diagnóstico e o tratamento de um câncer determinam um grande efeito na qualidade de vida dos indivíduos, a avaliação e a análise desses parâmetros tornaram-se uma preocupação da área médica ${ }^{7}$.

Até a década de 1970, o interesse pelo tema "qualidade de vida" se restringia às ciências humanas, sendo objeto de estudo de filósofos, cientistas sociais e políticos. A partir da década de 70, no contexto da transição epidemiológica e com o crescente interesse de dar "voz" aos pacientes, a expressão "qualidade de vida" (QV) passa a ser empregada na área da saúde costumeiramente associada à ausência de doença e ao bem-estar físico.

São várias as tentativas de delimitar o construto QV, sem, contudo, ter-se alcançado um consenso amplo. Isto se explica por sua complexidade, envolvendo fatores temporais, objetivos e subjetivos. A Organização Mundial de Saúde (OMS) define QV como "a percepção do indivíduo sobre sua posição na vida, no contexto da cultura e dos sistemas de valores nos quais ele vive, e em relação a seus objetivos, expectativas, padrões e preocupações”. . Nesta definição estão presentes a subjetividade, a multidimensionalidade e os aspectos positivos e negativos envolvidos no estado da pessoa. Embora haja divergências entre os diferentes pesquisadores a respeito do construto QV, esses três aspectos são consensuais nos estudos que tratam do tema.
Apesar de envolver subjetividades individuais e sistemas de significações culturais, na área da saúde, QV é geralmente mensurada na forma de questionários estruturados. Existem múltiplos questionários e os aspectos focados em cada um variam conforme os objetivos específicos dos estudos que os utilizam. Para tentar harmonizar essa situação, a Organização Mundial da Saúde estabeleceu um grupo de trabalho intitulado World Health Organization Quality of Life Group (WHOQOL Group), que desenvolveu um questionário, chamado WHOQOL-100, que considera vários aspectos em seis grandes domínios: (1) físico, (2) psicológico, (3) nível de independência, (4) relações sociais, (5) meio ambiente e (6) espiritualidade/religiosidade/crenças pessoais8.

$\mathrm{Na}$ oncologia, em um contexto no qual os indivíduos não mais morrem precocemente, porém têm uma sobrevida longa, são crescentes as preocupações com a QV que os pacientes terão nos anos seguintes ao diagnóstico e ao tratamento. Passou-se a valorizar parâmetros mais amplos do que simplesmente controle de sintomas, eficácia de tratamento, redução da mortalidade e aumento da expectativa de vida.

Em relação a pacientes com câncer da tireoide, apesar da sobrevida média ser normal, foi descrita diminuição na QV em longo prazo", com pacientes apresentando uma menor performance em áreas como vitalidade e desempenho emocional ${ }^{10}$. Deve-se ressaltar que um grande número de questionários estruturados utilizados para avaliar a QV de pacientes com PTC foram limitados pelo escopo das questões selecionadas e, assim, apresentaram um poder limitado de aferir as percepções e as experiências dos pacientes ${ }^{11} \mathrm{e}$ alguns temas relevantes podem não ter sido abordados $^{12}$. Revisão sistemática realizada em 2011 destacou que não há uniformidade na literatura quanto ao grau de prejuízo na QV desses indivíduos $^{13}$. Além disso, esse tema tem sido investigado por uma abordagem explanatória-causal, isto é, que objetiva identificar relações de causa e efeito; e não por uma aproximação hermenêutica, isto é, uma aproximação compreensivo-interpretativa de significados culturais e psicológicos. Pressupõe-se ser essencial, para o campo do cuidado à saúde, conhecer o que QV significa para os indivíduos, porque as pessoas estruturam e organizam suas vidas pessoais em torno dos significados atribuídos a situações ou objetos ${ }^{14}$.

Assim, o presente estudo objetivou abordar a QV de pacientes tratados com PTC sob a perspectiva dos próprios sujeitos, utilizando um método qualitativo de pesquisa em que a técnica 
de coleta de dados permitiu a livre expressão dos participantes, visando complementar, e de alguma forma superar, algumas limitações de estudos prévios que aplicaram instrumentos padronizados de avaliação.

\section{Metodologia}

Foi utilizado um método qualitativo aplicado a problemas clínicos ${ }^{14}$. Consistiu em coleta de dados por meio de entrevistas não diretivas com questões abertas, realizadas em 2010 e 2011, em uma amostra intencional, com definição do tamanho determinado por saturação teórica ${ }^{15-17}$, ou seja, quando se verificou que entrevistas adicionais passaram a não resultar em dados novos relevantes para a discussão teórica pretendida. Isso se verificou na avaliação dos pesquisadores a partir da décima quarta entrevista, tendo ainda sido feitas outras duas entrevistas para que esta constatação fosse confirmada. Consentimento informado foi obtido de todos os pacientes e o estudo foi aprovado pelo comitê de ética institucional.

A questão disparadora das entrevistas foi: "Como você avalia sua vida e seu cotidiano após o diagnóstico e o tratamento do seu câncer da tireoide?"

A livre expressão dos participantes do estudo foi estimulada, encorajando suas reflexões. Como alguns tópicos poderiam não ser espontaneamente mencionados, alguns subtemas foram ativamente apresentados aos entrevistados: Como foi para você saber o seu diagnóstico? Quais foram as repercussões do tratamento na sua vida? Como você vê a sua condição de saúde atual? Quais são os seus sentimentos a respeito da necessidade de tomar medicação de reposição tireoidiana continuamente? Como você se sente em relação à necessidade de realizar acompanhamento médico por toda a vida?

Foram entrevistados dezesseis pacientes com PTC tratado, 15 mulheres e um homem, com idade entre 18 e 45 anos. Todos os pacientes foram submetidos à tireoidectomia total, seguida por radioiodoterapia adjuvante (RAI), estavam em seguimento regular no serviço de Oncologia Cirúrgica da Santa Casa de São Carlos (São Paulo, Brasil), em tratamento com levotiroxina e não apresentavam sinais de doença metastática. Os sujeitos da pesquisa tinham diferentes graus de instrução e de poder aquisitivo e eram provenientes dos sistemas de saúde público e privado. As entrevistas foram transcritas literalmente e esse material empírico constituiu o "corpus da pesquisa".

O conteúdo do corpus foi analisado de forma independente por quatro pesquisadores (um estudante de medicina e três professores médi$\cos )$. Isto foi feito a partir de leituras atentas, o que possibilitou uma familiarização com este material e, em seguida, uma análise de conteúdo dos enunciados ali presentes. Individualmente, os pesquisadores elaboraram suas categorias analíticas, de acordo com a semelhança dos significados contidos nos enunciados proferidos pelos participantes ${ }^{18,19}$. Num momento seguinte, as convergências e as divergências destas análises individuais foram discutidas em grupo, chegando-se então a um consenso. Durante todo o processo, os pesquisadores tentaram manter uma postura de constante atenção aos detalhes e ao corpus como um todo, buscando a validade interna dos resultados, ou seja, procurando garantir que a categorização do material empírico representasse as vivências dos participantes. A partir disso, procurou-se elaborar uma discussão teórica, empiricamente fundamentada, sobre o tema qualidade de vida de pacientes com PTC.

\section{Resultados}

O Quadro 1 apresenta alguns dados sociodemográficos dos participantes.

De acordo com o método proposto, as entrevistas foram analisadas quanto aos enuncia$\operatorname{dos}^{19,20}$, tendo sido formuladas a partir destes sete categorias e 24 subcategorias analíticas de resultados (Quadro 2), correspondentes a núcleos de sentido identificados nas entrevistas organizando os elementos de significação úteis aos objetivos a que nos propusemos. Para cada uma das categorias citamos exemplos de enunciados que ajudam a exemplificá-las empiricamente.

\section{Experiências quanto ao prognóstico, incluindo a possibilidade de recidiva}

Foram obtidos relatos de desestabilização nas vidas dos pacientes entrevistados em função, sobretudo, do acompanhamento médico de longo prazo, particularmente nos momentos de realização dos "exames de controle" (é uma insegurança, morro de medo de acontecer de novo), mantendo por vezes um estado de constante alerta (tem que ficar sempre cuidando. Se acontecer de voltar, pelo menos pegaria no início, preciso me cuidar pra perceber logo se voltar). 
Quadro 1. Perfil sociodemográfico e clínico dos participantes da pesquisa.

\begin{tabular}{|c|c|}
\hline 1 & $\begin{array}{l}\text { Feminino; } 41 \text { anos; ensino médio; profissão antes e depois: balconista; branca; solteira; } 2 \text { filhos; católica. } \\
\text { Diagnóstico e cirurgia há } 9 \text { anos. Recorrência da doença há } 7 \text { anos, tratada com iodo radioativo. Iodo } \\
\text { radioativo há } 7 \text { e } 9 \text { anos. Última avaliação sem evidências clínicas e laboratoriais de recorrência da } \\
\text { doença. }\end{array}$ \\
\hline 2 & $\begin{array}{l}\text { Feminino; } 44 \text { anos; ensino fundamental incompleto; profissão antes: faxineira; profissão depois: } \\
\text { desempregada; parda; amasiada; } 2 \text { filhos; católica. Diagnóstico e cirurgia há } 5 \text { anos. Hipoparatireoidismo } \\
\text { durante } 2 \text { anos; quelóide em cicatriz cirúrgica. Iodo radioativo há } 4 \text { anos. Última avaliação sem evidências } \\
\text { clínicas e laboratoriais de recorrência da doença. }\end{array}$ \\
\hline 3 & $\begin{array}{l}\text { Feminino; } 35 \text { anos; ensino fundamental incompleto; profissão antes: empregada doméstica; profissão } \\
\text { depois: desempregada; branca; casada; } 3 \text { filhos; católica. Diagnóstico e cirurgia há } 8 \text { anos. Iodo radioativo } \\
\text { há } 8 \text { anos. Última avaliação sem evidências clínicas e laboratoriais de recorrência da doença. }\end{array}$ \\
\hline 4 & $\begin{array}{l}\text { Feminino; } 39 \text { anos; ensino médio; profissão antes: empregada doméstica; profissão depois: do lar; branca; } \\
\text { casada; } 2 \text { filhos; católica. Diagnóstico e cirurgia há } 11 \text { anos. Iodo radioativo há } 11 \text { anos. Última avaliação } \\
\text { sem evidências clínicas e laboratoriais de recorrência da doença. }\end{array}$ \\
\hline 5 & $\begin{array}{l}\text { Feminino; } 33 \text { anos; ensino médio; profissão antes: cabeleireira; profissão depois: está em afastamento; } \\
\text { branca; casada; } 1 \text { filho; evangélica. Diagnóstico e cirurgia há } 3 \text { anos. Recorrência da doença há } 1 \text { ano, } \\
\text { tratada com cirurgia. Iodo radioativo há } 2 \text { anos. Última avaliação sem evidências clínicas e laboratoriais } \\
\text { de recorrência da doença. }\end{array}$ \\
\hline 6 & $\begin{array}{l}\text { Feminino; } 43 \text { anos; ensino médio; profissão antes: caixa de supermercado; profissão depois } \\
\text { desempregada; branca; casada; } 1 \text { filho; evangélica. Diagnóstico e cirurgia há } 10 \text { anos. Recorrência da } \\
\text { doença há } 8 \text { anos, tratada com iodo radioativo. Iodo radioativo há } 8 \text { e } 10 \text { anos. Última avaliação sem } \\
\text { evidências clínicas e laboratoriais de recorrência da doença. }\end{array}$ \\
\hline 7 & $\begin{array}{l}\text { Feminino; } 39 \text { anos; ensino médio; profissão antes: operária da Eletrolux; profissão depois: desempregada; } \\
\text { branca; divorciada; } 1 \text { filho; evangélica. Diagnóstico e cirurgia há } 4 \text { anos. Iodo radioativo há } 4 \text { anos. Ưltima } \\
\text { avaliação sem evidências clínicas e laboratoriais de recorrência da doença. }\end{array}$ \\
\hline 8 & $\begin{array}{l}\text { Feminino; } 29 \text { anos; ensino superior; profissão antes: estudante; profissão depois: fisioterapeuta; branca; } \\
\text { solteira; sem filhos; espírita. Diagnóstico e cirurgia há } 6 \text { anos. Iodo radioativo há } 6 \text { anos. Última avaliação } \\
\text { sem evidências clínicas e laboratoriais de recorrência da doença. }\end{array}$ \\
\hline 9 & $\begin{array}{l}\text { Feminino; } 32 \text { anos; ensino médio; profissão antes: secretária; profissão depois: petshop; branca; solteira; } \\
1 \text { filho; católica. Diagnóstico e cirurgia há } 6 \text { anos. Iodo radioativo há } 6 \text { anos. Última avaliação sem } \\
\text { evidências clínicas e laboratoriais de recorrência da doença. }\end{array}$ \\
\hline
\end{tabular}

Foram observados relatos compatíveis com um processo de somatização e uma racionalização excessiva sobre a potencial gravidade da doença (isso é certo já: estou totalmente curada). Alguns negaram o problema, como se pode inferir a partir de enunciados de não querer saber de informações sobre a doença e seu prognóstico (não lembro se é benigno ou maligno). Por outro lado, houve referências à crença de fazer parte de um grupo positivamente diferenciado, por ter havido cura, e também a uma postura de altru- ísmo, aconselhando e orientando outras pessoas que passavam por experiências semelhantes.

As preocupações a respeito da necessidade de reposição hormonal medicamentosa durante toda a vida (dependo do remédio, aí acabou comigo) pareceram afetar negativamente alguns participantes, em razão da sensação de dependência contínua à medicação e do medo de experimentar novamente sintomas de hipotireoidismo, alguns parecendo sentir-se permanentemente a um passo de uma grave condição clínica (posso 
12 Feminino; 26 anos; profissão antes e depois: secretária; branca; casada; 1 filha. Diagnóstico há 6 anos, lobectomia há 6 anos e tireoidectomia total há 4 anos. Iodo radiativo há 4 anos. Última avaliação sem evidências clínicas e laboratoriais de recorrência da doença.

13 Feminino, 35 anos; ensino médio incompleto; profissão antes e profissão depois: sem trabalho; parda; casada; 1 filho; evangélica. Diagnóstico há 2 anos, cirurgia há 1 ano. Iodo radiativo a 2 meses. Última avaliação sem evidências clínicas e laboratoriais de recorrência da doença.

14 Feminino; 35 anos; ensino fundamental incompleto; profissão antes: cabeleireira; profissão depois: do lar; branca; amasiada; 2 filhos; evangélica. Diagnóstico e cirurgia há 12 anos. Iodo radiativo há 11 anos. Última avaliação sem evidências clínicas e laboratoriais de recorrência da doença.

15 Masculino; 37 anos; segundo grau completo; profissão antes e depois: montador de automóveis; branco; casado; 2 filhos; espírita. Diagnóstico e cirurgia há 6 anos. Iodo radiativo há 6 anos. Última avaliação sem evidências clínicas e laboratoriais de recorrência da doença.

16 Feminino; 29 anos; pós-graduada; profissão antes e depois: farmacêutica; branca; solteira; sem filhos; católica. Diagnóstico e cirurgia há 1 ano. Iodo radiativo há 6 meses. Última avaliação sem evidências clínicas e laboratoriais de recorrência da doença.

viver sem comida por um tempo, mas não sem a tiroxina).

\section{Impactos na vida social}

Os participantes referiram mudanças na dinâmica familiar e alterações de seus status sociais posteriormente à experiência com a doença e seu tratamento.

Alguns referiram abster-se do diálogo sobre a experiência do câncer para não causar preocupação aos familiares (difícil, chorava, não queria deixá-los preocupados, me trancava muito. Difícil chorar na frente das crianças, chorava sozinha mesmo). Em certos casos, foram percebidas marcantes mudanças nos costumes e hábitos da família (meus pais surtaram, tocavam violão todos os dias, era hábito dos dois; acabou, nunca mais). Alguns familiares teriam passado a atuar de forma ativa no seguimento da doença, pressionando o paciente a frequentar o serviço de saúde regularmente para realizar a rotina de controle do PTC (o marido fica falando: vê se é isso mesmo, se é normal).
Houve relatos de fortes impactos na vida social, como a percepção de preconceito relacionado ao diagnóstico (algumas pessoas ficavam preocupadas de estar perto de mim e pegarem a doença), incluindo dentro da rede de amigos. Foram percebidas mudanças do status social pela associação da imagem do indivíduo à de um paciente terminal (a pessoa chega com aquela cara de dó, como se você estivesse morrendo, é horrível).

\section{Alterações na percepção da imagem corporal}

Foram descritos sintomas físicos tardios relacionados, na visão dos entrevistados, ao tratamento recebido (tireoidectomia e terapia com iodo radioativo). Referiram esses sintomas como importantes, mesmo acreditando serem de causa psicológica, pois não foi encontrada uma explicação fisiopatológica para estes (fiz o exame, não tem nada, ele falou que é psicológico mesmo). Foram mencionados, por exemplo, dor no local da cirurgia depois de anos do procedimento, dores musculares gerais, cansaço, perda de libido, difi- 
Quadro 2. Categorização analítica dos dados coletados.

\begin{tabular}{|c|}
\hline Categorias de resultados \\
\hline $\begin{array}{l}\text { 1. Experiências relacionadas ao prognóstico da } \\
\text { doença e à possibilidade de recidiva da doença ao } \\
\text { longo da vida. } \\
\text { Desestabilização periódica em função dos exames } \\
\text { "de controle" } \\
\text { Estado de alerta } \\
\text { Mobilização de mecanismos de defesa psicológica } \\
\text { Mecanismos de superação } \\
\text { Ser uma hipotireoidea em potencial }\end{array}$ \\
\hline $\begin{array}{l}\text { 2. Impacto na vida social. } \\
\text { Preocupação com os familiares } \\
\text { Alteração dos hábitos familiares } \\
\text { Preocupação dos familiares } \\
\text { Percepção de preconceito e de mudanças negativas } \\
\text { no status social }\end{array}$ \\
\hline $\begin{array}{l}\text { 3. Percepção corporal alterada. } \\
\text { Conscientização do corpo } \\
\text { Sintomas sem causas biológicas identificadas } \\
\text { Sequelas cosméticas presentes } \\
\text { Sequelas biológicas }\end{array}$ \\
\hline $\begin{array}{l}\text { 4.Manejo da ideia de causalidade do câncer } \\
\text { Atribuição da causalidade do câncer } \\
\text { Genética, radiação e produtos químicos como riscos } \\
\text { modernos ("fatores do câncer") } \\
\text { Pressões extras "anticâncer" } \\
\text { Busca de informações }\end{array}$ \\
\hline $\begin{array}{l}\text { 5. Revisão positiva de alguns conceitos de vida } \\
\text { pessoais. } \\
\text { Percepção de uma quebra } \\
\text { Religiosidade Revista } \\
\text { Vivência de uma provação }\end{array}$ \\
\hline $\begin{array}{l}\text { 6. Inserção na semântica médica } \\
\text { Tentativa de apropriação/tradução do conhecimento } \\
\text { médico } \\
\text { Postura crítica em relação aos serviços de saúde }\end{array}$ \\
\hline $\begin{array}{l}\text { 7. Impacto no trabalho } \\
\text { Mudança de trabalho } \\
\text { Interrupção do trabalho devido às "sequelas" }\end{array}$ \\
\hline
\end{tabular}

culdade para perder peso e dor vaga na região do pescoço.

A presença de sequela cirúrgica objetiva, como rouquidão, também foi mencionada (se fico ansiosa, nervosa, fico rouca, minha voz até some). Não foram relatadas outras possíveis complicações dos tratamentos citadas na litera- tura médica (hipocalcemia persistente, alterações de deglutição, alteração no paladar, diminuição da lubrificação ocular e problemas dentais).

Também não referiram que a sequela cosmética da cirurgia (cicatriz) tivesse efeitos negativos (tudo certo, até a cicatrização, se você olhar não tenho nada). Objetivamente, nenhum dos pacientes desse estudo tinha cicatriz muito evidente e todos se compararam positivamente a pacientes que sofreram a mesma cirurgia, o que pode ter influenciado sua postura positiva em relação à cicatriz cirúrgica (cicatriz arrasou [isto é, ficou ótima]).

\section{Manejo conceitual da etiologia do câncer}

Foi observado, na fala dos participantes, a imputação de uma gama de causas para o câncer: causas presumidas, não controláveis e não totalmente compreendidas (onde morava teve muito problema de iodo, achava que tinha pego algum problema de lá: da alimentação, da água, algo assim).

Alguns relataram que o PTC poderia ter decorrido de fatores emocionais (estresse, sofrimento na infância, culpas por eventos da vida pessoal) (acumulei arrependimentos e maus sentimentos e acho que desenvolvi o câncer por isso) ou mesmo por ter simplesmente pronunciado a palavra "câncer"; a autopiedade foi mencionada como fator de piora do prognóstico da doença. Outros relataram ter sido apenas uma fatalidade (má sorte) ou que o surgimento do câncer foi influenciado por fatores genéticos (algo meio que genético, né?, fizeram uma pesquisa sobre a doença que tenho, é hereditário), radiação (acho que pode ter por fator emocional, radiação também) por uso de determinados produtos químicos (mexia com produtos químicos fortíssimos então não sei se teve influência).

Relataram, por outro lado, mudanças de hábitos vistos como prejudiciais à saúde e oncogênicos, como o fumo, as emoções negativas e a alimentação considerada pouco saudável. As participantes expressaram percepção de uma pressão social anticâncer em suas vidas diárias, resultando na mudança de hábitos por parte de algumas delas (sempre com o filtro solar, sempre passando).

Embora alguns tenham referido que o médico é a pessoa mais capaz para lhes fornecer informações e avaliar o prognóstico da doença, outros buscaram fontes de informações alternativas, como a opinião de outras pessoas e a internet (pesquiso pela internet, coisas que desconheço, até o tratamento, pra saber se tem algo novo). 


\section{Revisão positiva de alguns conceitos de vida pessoais}

Alguns participantes relataram mudanças positivas em suas personalidades e visões de mundo. Ocorreu para alguns entrevistados uma revisão no sentido da vida, tendo a vivência do câncer sido vista como uma "quebra" (mudei, me tornei bem mais flexível, marca a sua vida de forma muito profunda, uma experiência muito profunda tanto física quanto psíquica), a partir da qual a vida dos pacientes sofreu mudanças significativas, como passar a morar fora da casa da família nuclear, retomar planos de estudo, levar a vida mais “zen" e tornar-se uma pessoa "melhor".

Houve relatos de revisão da religiosidade, com mudança de religião ou maior apego religioso (você fica mais próxima de Deus, me superei bastante, me entreguei a Jesus). Os pacientes referiram ter obtido uma vitória pessoal, por terem sido capazes de vencer um obstáculo importante e se recuperarem (tinha que passar por isso, cada um tem sua cruz para carregar, se Deus permitiu isso na minha vida, algum propósito tem, que seja a vontade de Deus, uma prova).

\section{Inserção na semântica médica}

Os participantes deram indícios de que passaram a utilizar mais os termos médicos nas suas falas e a incorporar conceitos médicos (não foi por causa do cigarro, porque os fatores [de risco] do câncer da tireoide não é ser fumante, mas tenho que parar porque a primeira metástase que dá é no pulmão). Observamos tentativas de explicações de quadros clínicos com termos técnicos e procura de explicações fisiopatológicas para seus problemas de saúde (só tive um pouquinho de dor nas parótidas, mas não sabia se era ou não do iodo, porque fazia um ano que tinha tomado o iodo). Passaram também a ser mais críticos em relação aos serviços de saúde, refletindo sobre a qualidade destes, dos profissionais e do cuidado (tudo certo lá, tudo superorganizado; voltava no posto, fazia exame e nada, até que procurei outro médico particular, fui, paguei a consulta e fiz os exames; aí marquei consulta com um outro médico, ele me examinou tudo certinho e pediu pra fazer alguns exames).

\section{Impacto no trabalho}

Participantes referiram mudanças profissionais, assumindo trabalhos menos qualificados ou informais (trabalhei durante dez anos numa fabrica. Fiquei afastada um ano e fui trabalhar com minha mãe). Aparentemente perceberam novos desafios em seus trabalhos antigos, em consequência da doença, seu tratamento ou suas sequelas, reais ou não, que em alguns casos os levou a abandonar a antiga ocupação (fiquei bem sensivel a produtos químicos. não posso perceber os cheiros. Então parei com isso sabe). Houve relato de identificação de supostos fatores patogênicos no antigo trabalho e de situações em que foi interrompida a vida laboral (depois do diagnóstico, pedi demissão do emprego e não trabalho mais), mesmo que temporariamente (no momento, não trabalho por causa dos exames).

\section{Discussão}

Embora as sete categorias analíticas de resultados pareçam revelar uma influência estressante do PTC na vida dos participantes, é necessário enfatizar que as narrativas também indicam alguns pontos positivos dessa vivência, como, por exemplo, mecanismos de superação que se desenvolveram após a experiência com a patologia e seu tratamento - como o altruísmo - , mudanças para hábitos de vida mais saudáveis, suporte familiar recebido ao longo do processo e revisão de alguns conceitos de vida pessoais.

No entanto, os relatos foram permeados mais intensamente por referências a problemas e dificuldades, o que permite supor uma persistência de inquietações que prejudicam a qualidade de vida dos participantes. Isto, a despeito de que, na amostra aqui estudada, o tempo médio desde o diagnóstico de PTC foi de 7,8 anos (variando entre 3 e 16 anos), ou seja, quando já se poderia supor que as repercussões tivessem sido supera$\operatorname{das}^{21,22}$.

A seguir, procuramos discutir e interpretar as categorias analíticas dos resultados. Primeiramente, dialogamos com a literatura sobre QV de pacientes com PTC. Em seguida, duas outras chaves interpretativas adicionais abordam pontos que consideramos relevantes para os pacientes, mas que não são discutidos nos estudos da qualidade de vida naqueles com câncer de tireoide: a contraposição de dois universos semânticos relacionados à saúde (popular vs médico) e a questão de uma possível caracterização do PTC como um risco moderno. 


\section{Experiência com o PTC e a qualidade de vida}

Os resultados a que chegamos vão ao encontro de estudos que concluíram que, apesar da melhora na QV depois do primeiro ano desde o diagnóstico de PTC, pode não haver uma normalização em longo $\mathrm{prazo}^{22}$. Algumas subpopulações de pacientes com PTC continuaram, mesmo depois de anos, relatando significativamente mais problemas, como insônia, de voz e estresse ${ }^{23}$. Outros estudos mostraram que sobreviventes de câncer de tireoide frequentemente vivenciam mais problemas do que os indivíduos controle, independente de idade e gênero, e que a QV nem sempre está diretamente relacionada à gravidade do prognóstico ${ }^{24}$. Schultz et al. ${ }^{25}$ mostraram que os pacientes curados de câncer de tireoide relataram perda de memória e mais problemas psicológicos do que sobreviventes de outros tipos de câncer. Outra pesquisa concluiu que os parâmetros de saúde física e mental de pacientes com PTC tratados em longo prazo não foram significativamente diferentes da população controle ${ }^{26}$, enquanto alguns estudos transversais concluíram não haver associação significativa entre QV e tempo de doença ${ }^{27}$. Por outro lado, o trabalho de Miccoli et al. ${ }^{28}$ mostrou que o procedimento da tireoidectomia, consequente a diversas patologias tireoidianas, influencia por si só na QV, com piores índices globais e particular piora na função social, assim como foi demonstrado para outros procedimentos cirúrgicos ${ }^{29}$. Observa-se, portanto, ainda uma grande instabilidade das hipóteses que tentam explicar a questão da $\mathrm{QV}$ de pacientes com PTC, permanecendo pouco definido o que mais exatamente poderia a estar influenciando a QV.

Entre os participantes entrevistados, houve referências contundentes a alterações na vida sociofamiliar, o que contrasta com o que foi encontrado por Tan et al..$^{30}$, em estudo com 152 indivíduos asiáticos, que, apenas para a "função social", não constatou alterações da QV. Inversamente, nossos resultados ratificam os de outras pesquisas anteriores e apontam inclusive para a possibilidade de ocorrência do fenômeno da estigmatização social de pacientes com PTC. Isto foi percebido pelos participantes, embora seja algo pouco esperado, dado o bom prognóstico e a quase ausência de sequelas cirúrgicas visíveis.

$\mathrm{Na}$ dimensão social, sabe-se que outro aspecto capaz de influenciar a QV dos indivíduos é a mudança em sua vida laboral ${ }^{10}$. Alguns de nossos entrevistados referiram, de fato, uma profunda desestabilização desta dimensão de suas vidas.
A situação traumática representada pelo momento do diagnóstico ${ }^{27}$ parece gerar um estado de ansiedade de alerta constante de alguns pacientes, que frequentemente aparentam reviver sentimentos dessa época. Alguns choraram durante as entrevistas, mostrando talvez que certos aspectos da experiência ainda não estavam psicologicamente superados, revelando temor de reviver a experiência negativa. Isto parece estar relacionado, sobretudo, à ansiedade antecipatória com vistas à perspectiva de uma recidiva que, mesmo sem ter sido constatada entre os participantes, permanece como uma possibilidade. Secundariamente, pode estar também ligada à quebra da rotina necessária para viabilizar os exames de seguimento do tumor.

Para lidar com as angústias relacionadas à doença e seu tratamento, alguns participantes mobilizaram, marcantemente, certos mecanismos de defesa psicológica, geralmente considerados adequados para o processo de superação de problema de saúde (como o altruísmo), mas também outros considerados a priori como pouco saudáveis (como a negação).

No manejo das repercussões objetivas e subjetivas do PTC e seu tratamento, os participantes mencionaram valorizar os suportes social, familiar e dos profissionais de saúde para a superação dos problemas que enfrentaram, entre eles o medo de recidiva, algo também observado por Sawka et al. ${ }^{12}$. Mencionaram também o incômodo com a dependência de medicamentos em longo prazo e com o fato de ser uma pessoa hipotireoidea em potencial, fenômenos também relatados por Botella-Carretero et al. ${ }^{31}$.

\section{Aproximação de dois universos semânticos}

A experiência dos participantes com o PTC os teria levado a uma necessidade subjetiva de se aproximar e de se apropriar de conhecimentos médicos. Isto ocorreu em específico, por exemplo, em relação à questão envolvida na oposição conceitual entre malignidade versus benignidade. Essa tentativa de apropriação dos conceitos médicos teria sido expandida a outros aspectos da área da saúde, e alguns pacientes inclusive passaram a adotar uma postura mais crítica em relação à prática médica e ao sistema de saúde.

Embora a "oportunidade de adquirir novas informações e habilidades" seja um subdomínio já previsto em alguns questionários estruturados de QV, a questão específica das dificuldades de inserção no universo semântico médico não é abordada pelos atuais instrumentos de avaliação 
de QV, deixando de lado uma questão de alta relevância para alguns pacientes, sobretudo talvez para os acometidos por doenças potencialmente letais.

Sawka et al. ${ }^{12}$ constataram que, para os pacientes do seu estudo, a opinião do médico sobre sua condição clínica e sobre o tratamento foi altamente valorizada. Isto foi ratificado em nossa amostra, porém verificamos também que alguns participantes procuram inclusive por signos (comportamentos) não verbais que indicassem seu estado de saúde. Em seu estudo, Sawka et al. ${ }^{12}$ também relatou que a internet foi procurada como meio de informação pelos participantes, pois é uma fonte de informação mais acessível, como mostraram os estudos de Roberts et al. ${ }^{32}$, porém as informações contidas nesse meio não foram consideradas relevantes pelos pacientes para o entendimento da sua doença e da sua condição de vida específica.

Embora o médico tenha permanecido, na perspectiva dos participantes do presente estudo, como o principal intérprete de suas condições de saúde e dos riscos a que estão submetidos, cremos ter identificado alguns possíveis ruídos, e mesmo obstáculos, neste processo de comunicação e tradução desse conhecimento. Parece haver uma constante tensão neste processo, ao envolver a aproximação de universos conceituais muito díspares, um deles altamente reificado pela produção científica (medicina) e outro fundamentado no senso comum (conhecimento prévio dos pacientes), utilizando a terminologia da teoria das representações sociais.

\section{A experiência do câncer da tireoide como uma preocupação de risco moderno}

A informação adquirida a respeito do próprio corpo, durante o processo de diagnóstico e tratamento, "mal interpretada" ou não, teria aumentado o estado de alerta quanto à própria saúde e a preocupação e a vigilância dos pacientes em relação ao seu próprio corpo, numa tentativa de detectar precocemente uma recidiva do câncer e/ ou suas sequelas biológicas e não biológicas. Adicionalmente, a aquisição de conhecimento médico, através da informação adquirida diretamente do profissional de saúde ou mesmo da internet, pareceram levar os pacientes a elaborarem explanações etiofisiopatológicas populares/informais a respeito do seu câncer e de outros problemas de saúde não relacionados. Dependendo do conhecimento que tinham sobre a doença e da compreensão das informações médicas às quais tiveram acesso, os indivíduos desenvolveram ideias peculiares sobre a causalidade da doença que tiveram.

Observamos que, no presente trabalho, todos os pacientes entrevistados receberam o mesmo tratamento. Como relatado por Buick ${ }^{33}$, pode existir uma influência do tipo de tratamento realizado sobre as diferentes visões de causalidade, algo sugerido quando o autor comparou as visões de pacientes com câncer de mama que receberam quimioterapia ou radioterapia. Os que receberam quimioterapia atribuíram a causa do câncer a fatores de riscos comportamentais e ambientais e os que receberam radioterapia atribuíram sua patologia a uma fatalidade.

Diferentes compreensões e hipóteses causais da doença também podem estar relacionadas com a escolaridade do paciente, tendo um menor grau de instrução sido relacionado negativamente com a percepção medicamente adequada da doença, afetando a $\mathrm{QV}^{9}$. Por exemplo, concepções muito díspares dos atuais conhecimentos epidemiológicos poderiam determinar alterações importantes de hábitos de vida, para evitar fatores que foram inadequadamente atribuídos como causais. Assim, observe-se que, além da variável "conhecimentos prévios", há indícios de que diferentes tipos e intensidades de exposição a tecnologias médicas promovem diferentes reações emocionais e elaborações cognitivas visando compreender a causalidade e o risco.

O constrangimento frente aos saberes médicos aos quais os pacientes foram instados a se introduzir, particularmente no sentido de interpretar a (suposta) causalidade do câncer, faz pensar que esta experiência configura-se parcialmente como uma preocupação sobre o "risco moderno", à medida que os participantes não se perceberam dispondo dos "órgãos sensoriais" das ciências médicas para interpretar as ameaças que os intimidam $^{34}$. Tais aspectos também não são abordados nos atuais questionários estruturados de QV.

A proposta de construir uma teoria da sociedade global de risco é apresentada pelo sociólogo alemão Ulrich Beck ${ }^{34}$, que defende que houve uma ruptura dentro da modernidade que a afastou da sociedade industrial clássica, caracterizada pela produção e distribuição de bens, e fez surgir algo diferente: a sociedade do risco. Para $\mathrm{Beck}^{34}$, a noção de risco toma um significado bastante específico e se baseia em interpretações causais dos acontecimentos. Os riscos modernos permanecem invisíveis, pois só se estabelecem a partir dos saberes. O centro da consciência de risco reside em projeções para o futuro e não no presente, o que pressupõe um processo social de reconheci- 
mento e legitimação. Na construção desta consciência de risco a ciência é um dos mais fundamentais meios de legitimação e reconhecimento dos $\operatorname{riscos}{ }^{34}$.

\section{Limitações do estudo}

A seleção de participantes de amostras intencionais, típicas das pesquisas qualitativas, implica na discussão dos possíveis vieses determinados por ela. A generalização dos presentes achados pode ser limitada pelas características demográficas da amostra, em que há um predomínio absoluto de mulheres entre os participantes, o que reflete, porém, a distribuição entre gêneros do agravo à saúde em foco. Todos os pacientes foram submetidos à tireoidectomia total e RAI, o que pode indicar uma maior complexidade na patologia dos participantes, uma vez que estes foram recrutados num centro terciário de atenção à saúde. Os pacientes foram operados por um único cirurgião e isto poderia afetar a incidência de sequelas cirúrgicas e o vínculo do paciente com o serviço médico. A outra limitação é intrínseca das pesquisas qualitativas e diz respeito ao repertório interpretativo dos pesquisadores, que influencia inclusive na saturação teórica dos resultados.

Em relação às diferenças de sexo e idade, estudos mostram que pacientes mais jovens apresentam um menor comprometimento na QV, inclusive sem nenhum na fase de adolescência ${ }^{35}$, enquanto mulheres referem mais problemas do que homens $s^{10,36}$, independente do fato da incidência de câncer de tireoide ser maior nelas do que neles. Isto não pôde ser inferido em nosso trabalho, pois nossa população amostral foi restrita a adultos jovens, com maioria de pacientes do sexo feminino.

\section{Conclusões}

Os desenvolvimentos de questionários estruturados de medição de QV, com a definição dos domínios e subdomínios relevantes para a saúde das pessoas, passam necessariamente por estudos compreensivos-interpretativos (ou qualitativos). No caso, por exemplo, do desenvolvimento do WHOQOL-100, procurou-se inclusive circunscrever um "universal cultural" de QV. No entanto, a dinamicidade dos significados histórico-culturais e psicológicos em saúde, hoje em dia constantemente influenciados por inovações tecnológicas e por interpretações epidemiológicas que se sucedem, requer estudos qualitativos que captem mudanças nos aspectos subjetivos que subsidiam o construto.

Constatamos que a experiência de ter sido diagnosticado e tratado para PTC, levou a mudanças positivas e negativas na qualidade de vida dos participantes e que a utilização de um método qualitativo aplicado a problemas clínicos permitiu a análise e a interpretação dos significados dessas experiências (diagnostico e tratamento do PTC) e suas representações, levando à identificação de possíveis determinantes da QV de adultos jovens com PTC tratado.

As categorias de resultados a que chegamos através da análise das expressões livres dos participantes, incitadas por entrevistas semiestruturadas, certamente contêm aspectos já previstos em questionários como o WHOQOL. Porém, outros aspectos nos parecem estar apenas parcialmente previstos, como o manejo do conceito de etiologia de uma doença e a questão da inserção "forçada" dos pacientes ao universo conceitual da medicina. Adicionalmente, a interpretação que se pôde dar a alguns destes aspectos beneficiou-se de elaborações teóricas de desenvolvimento mais recente, como a questão das angústias quanto ao surgimento ou à recidiva do PTC configuraremse subjetivamente para os participantes como um "risco moderno", no contexto de uma "sociedade de riscos”. 


\section{Colaboradores}

CMR Germano, D Bonato, VH Maion, LRS Avó, DG Melo e BJB Fontanella participaram da redação final do artigo e concordam com a versão enviada.

\section{Agradecimentos}

A pesquisa recebeu financiamento do CNPQ na forma de uma bolsa de Iniciação Científica para Débora Bonato.

\section{Referências}

1. Agate L, Lorusso L, Elisei R. New and old knowledge on differentiated thyroid cancer epidemiology and risk factors. J Endocrinol Invest 2012; 35(Supl. 6):3-9.

2. Veiga LH, Neta G, Aschebrook-Kilfoy B, Ron E, Devesa SS. Thyroid cancer incidence patterns in São Paulo, Brazil and the U.S. SEER program, 1997-2008. Thyroid 2013; 23(6):748-757.

3. Aschebrook-Kilfoy B, Ward MH, Sabra MM, Devesa SS. Thyroid cancer incidence patterns in the United States by histologic type, 1992-2006. Thyroid 2011; 21(2):125-134.

4. RH, Kaplan SP, Cao H, Weiss RE, Degroot LJ, Simon CA, Embia OM, Angelos P, Kaplan EL, Schechter RB. A study of recurrence and death from papillary thyroid cancer with 27 years of median follow-up. Surgery 2013; 154(6):1436-1446.

5. Pacini F, Castagna MG, Brilli L, Pentheroudakis G; ESMO Guidelines Working Group. Thyroid cancer: ESMO Clinical Practice Guidelines for diagnosis, treatment and follow-up. Ann Oncol 2012; Supl. 7:vii110119.

6. Verburg FA, Mäder U, Tanase K, Thies ED, Diessl S, Buck AK, Luster M, Reiners C. Life expectancy is reduced in differentiated thyroid cancer patients $\geq 45$ years old with extensive local tumor invasion, lateral lymph node, or distant metastases at diagnosis and normal in all other DTC patients. J Clin Endocrinol Metab 2013; 98(1):172-180.

7. Mostoufi-Moab S, Barakat LP, Bauer AJ. Quality of life in adolescent patients with differentiated thyroid cancer: moving beyond survival. J Clin Endocrinol Metab 2012; 97(10):3453-3456.

8. Study protocol for the World Health Organization project to develop a Quality of Life assessment instrument (WHOQOL). Qual Life Res 1993;2(2):153-159.

9. Singer S, Lincke T, Gamper E, Bhaskaran K, Schreiber S, Hinz A, Schulte T. Quality of life in patients with thyroid cancer compared with the general population. Thyroid 2012; 22(2):117-124.

10. Crevenna R, Zettinig G, Keilani M, Posch M, Schmidinger $M$, Pirich C, Nuhr M, Wolzt M, Quittan M, Fialka-Moser V, Dudczak R. Quality of life in patients with non-metastatic differentiated thyroid cancer under thyroxine supplementation therapy. Support Care Cancer 2003; 11(9):597-603.

11. Vissers PA, Thong MS, Pouwer F, Zanders MM, Coebergh JW, van de Poll-Franse LV. The impact of comorbidity on Health-Related Quality of Life among cancer survivors: analyses of data from the PROFILES registry. J Cancer Surviv 2013; 7(4):602-613.

12. Sawka AM, Goldstein DP, Brierley JD, Tsang RW, Rotstein L, Ezzat S, Straus S, George SR, Abbey S, Rodin G, O’Brien MA, Gafni A, Thabane L, Goguen J, Naeem A, Magalhaes L. The impact of thyroid cancer and pos$\mathrm{t}$-surgical radioactive iodine treatment on the lives of thyroid cancer survivors: a qualitative study. PLoS One 2009; 4(1):e4191.

13. Husson O, Haak HR, Oranje WA, Mols F, Reemst PH, van de Poll-Franse LV. Health-related quality of life among thyroid cancer survivors: a systematic review. Clin Endocrinol 2011; 75(4):544-554. 
14. Turato ER. Tratado da metodologia da pesquisa clínicoqualitativa: construção teórico-epistemológica, discussão comparada e aplicação nas áreas de saúde e humanas. $4^{\mathrm{a}}$ ed. Petrópolis: Vozes; 2010.

15. Glaser BG, Strauss AL. The Discovery of Grounded Theory: Strategies for Qualitative Research. Chicago: Aldine Publishing Company; 1967.

16. Fontanella BJB, Magdaleno Júnior R. Saturação teórica em pesquisas qualitativas: contribuições psicanalíticas. Psicologia em Estudo 2012, 17(1):63-71.

17. Fontanella BJB, Luchesi BM, Saidel MG, Ricas J, Turato ER, Melo DG. Sampling in qualitative research: a proposal for procedures to detect theoretical saturation. Cad Saude Publica 2011; 27(2):388-394.

18. Minayo MCS. O desafio do conhecimento: pesquisa qualitativa em saúde. 14a Ed. São Paulo: Hucitec; 2014.

19. Bardin L. Análise de conteúdo. São Paulo: Editora 70; 2011.

20. Minayo MCS. Qualitative analysis: theory, steps and reliability. Cien Saude Colet 2012; 17(3):621-626.

21. Kent EE, Ambs A, Mitchell SA, Clauser SB, Smith AW, Hays RD. Health-related quality of life in older adult survivors of selected cancers: data from the SEER -MHOS linkage. Cancer 2015; 121(5):758-765.

22. Husson O, Haak HR, Buffart LM, Nieuwlaat WA, Oranje WA, Mols F, Kuijpens JL, Coebergh JW, van de Poll-Franse LV. Health-related quality of life and disease specific symptoms among (long-term) thyroid cancer survivors: a study from the population-based PROFILES registry. Acta Oncol 2013; 52(2):447-454.

23. Roerink SH, de Ridder M, Prins J, Huijbers A, de Wilt HJ, Marres H, Repping-Wuts H, Stikkelbroeck NM, Timmers HJ, Hermus AR, Netea-Maier RT. High level of distress in long-term survivors of thyroid carcinoma: results of rapid screening using the distress thermometer. Acta Oncol 2013; 52(1):128-137.

24. Sawka AM, Naeem A, Jones J, Lowe J, Segal P, Goguen J, Gilbert J, Zahedi A, Kelly C, Ezzat S. Persistent posttreatment fatigue in thyroid cancer survivors: a scoping review. Endocrinol Metab Clin North Am 2014; 43(2):475-494.

25. Schultz PN, Stava C, Vassilopoulou-Sellin R. Health profiles and quality of life of 518 survivors of thyroid cancer. Head Neck 2003; 25(5):349-356.

26. Hoftijzer HC, Heemstra KA, Corssmit EP, van der Klaauw AA, Romijn JA, Smit JW. Quality of life in cured patients with differentiated thyroid carcinoma. J Clin Endocrinol Metab 2008; 93(1):200-3.

27. Tagay S, Herpertz S, Langkafel M, Erim Y, Bockisch A, Senf W, Görges R. Health-related Quality of Life, depression and anxiety in thyroid cancer patients. Qual Life Res 2006; 15(4):695-703.
28. Miccoli P, Minuto MN, Paggini R, Rucci P, Oppo A, Donatini G, Golia F, Novelli L, Carlini M, Dell'Osso L. The impact of thyroidectomy on psychiatric symptoms and quality of life. J Endocrinol Invest 2007; 30(10):853-859.

29. Bezerra KB, Silva DSM, Chein MBC, Ferreira PR, Maranhão JKP, Ribeiro NL, Mochel EG. Qualidade de vida de mulheres tratadas de câncer de mama em uma cidade do nordeste do Brasil. Cien Saude Colet 2013; 18(7):1933-1941.

30. Tan LG, Nan L, Thumboo J, Sundram F, Tan LK. Health-related quality of life in thyroid cancer survivors. Laryngoscope 2007; 117(3):507-510.

31. Botella-Carretero JI, Galán JM, Caballero C, Sancho J, Escobar-Morreale HF. Quality of life and psychometric functionality in patients with differentiated thyroid carcinoma. Endocr Relat Cancer 2003; 10(4):601-610.

32. Roberts KJ, Lepore SJ, Urken ML. Quality of life after thyroid cancer: an assessment of patient needs and preferences for information and support. $J$ Cancer Educ 2008; 23(3):186-191.

33. Buick DL. Illness representations and breast cancer: coping with radiation and chemotherapy. In: Petrie KJ, Weinman J, editors. Perceptions of Health and Illness. Amsterdam: Harwood Academic; 1997. p. 379-409.

34. Beck U. Sociedade de risco: rumo a uma outra modernidade. São Paulo: Editora 34; 2011.

35. Oren A, Benoit MA, Murphy A, Schulte F, Hamilton J. Quality of life and anxiety in adolescents with differentiated thyroid cancer. J Clin Endocrinol Metab 2012; 97(10):E1933-1937.

36. Giusti M, Melle G, Fenocchio M, Mortara L, Cecoli F, Caorsi V, Ferone D, Minuto F, Rasore E. Five-year longitudinal evaluation of quality of life in a cohort of patients with differentiated thyroid carcinoma. J Zhejiang Univ Sci B 2011; 12(3):163-173.

Artigo apresentado em 09/03/2015

Aprovado em 16/11/2015

Versão final apresentada em 18/11/2015 